\title{
Bulimia nervosa in adolescents: prevalence and treatment challenges
}

This article was published in the following Dove Press journal:

Adolescent Health, Medicine and Therapeutics

\section{Lisa Hail \\ Daniel Le Grange}

Department of Psychiatry, University of California, San Francisco, CA, USA

Correspondence: Lisa Hail

Department of Psychiatry, University of California, 40I Parnassus Avenue,

Box 0984, San Francisco, CA 94I43,

USA

Tel +I 4I5 4767857

Email Lisa.Hail@ucsf.edu

\begin{abstract}
Bulimia nervosa $(\mathrm{BN})$ is a serious psychiatric illness that typically develops during adolescence or young adulthood, rendering adolescents a target for early intervention. Despite the increasing research devoted to the treatment of youth with anorexia nervosa (AN) and adults with $\mathrm{BN}$, there remains a dearth of evidence for treating younger individuals with BN. To date, there have been four published randomized controlled trials comparing psychosocial treatments, leaving significant room to improve treatment outcomes. Family-based treatment is the leading treatment for youth with $\mathrm{AN}$, while cognitive-behavioral therapy is the leading intervention for adults with $\mathrm{BN}$. Involving caregivers in treatment shows promising results, however, additional research is needed to investigate ways in which this treatment can be adapted further to achieve higher rates of recovery. Keywords: eating disorders, youth, family-based treatment, cognitive behavioral therapy, psychodynamic therapy, psychopharmacological treatment, evidence based treatment
\end{abstract}

\section{Introduction}

Eating disorders (EDs) are serious psychiatric illnesses that typically develop during adolescence or young adulthood. These disorders are associated with both physical and psychological sequelae and often lead to considerable impairment and distress. ${ }^{1}$ The fifth edition of the Diagnostic and Statistical Manual of Mental Disorders (DSM) ${ }^{2}$ recognizes eight feeding disorders and EDs, including bulimia nervosa (BN), anorexia nervosa (AN), and subthreshold presentations that are classified under unspecified feeding or ED. While the field has advanced the understanding of treatment approaches for adolescents with $\mathrm{AN}$ and adults with $\mathrm{BN}$ in particular, evidence to guide the treatment of $\mathrm{BN}$ in youth lags behind.

\section{Diagnosis of BN}

BN is characterized by recurrent episodes of eating an objectively large amount of food (ie, binge) with an associated loss of control, as well as inappropriate compensatory behavior (eg, self-induced vomiting, misuse of laxatives or diuretics, fasting, or excessive exercise) and overvaluation of shape and weight. ${ }^{2}$ In the current edition of the DSM, these episodes of binge eating and compensatory behavior must occur at a minimum frequency of once per week over the course of 3 months. In the DSMIV-TR, the minimum frequency of these episodes of binge eating and compensatory behavior was double (ie, an average of twice per week). ${ }^{3}$ In studies comparing full and subthreshold presentations of DSM-IV BN, those individuals who were subthreshold reported significantly more subjective binge episodes in which they experienced a 
loss of control but did not consume an objectively large portion of food. ${ }^{4}$ Purge frequency across both groups was similar, suggesting that the experience of losing control may be more salient than the amount of food consumed. ${ }^{4}$ Diagnostic crossover is relatively common, especially among youths, with many who initially present with subthreshold presentations progressing to meet full diagnostic criteria for BN or less commonly AN. ${ }^{4,5}$ This diagnostic fluidity suggests that clinical management should not differ between sub- and full-threshold BN.

Traditional diagnostic approaches rely on the self-report of symptoms by the patient. While the low body weight characteristic of AN makes it more outwardly visible, $\mathrm{AN}$ is considered an ego-syntonic illness, such that it is consistent with the self-concept of the sufferer, making them less open to treatment. ${ }^{5}$ Conversely, $\mathrm{BN}$ is considered ego-dystonic and aversive to the patient, yet shame and secrecy often inhibit disclosure of symptoms and seeking treatment. In younger populations, developmental concerns, such as the ability to endorse such conceptual symptoms as "undue influence of weight and shape" may further impact the ability to diagnose full-threshold EDs in adolescents. ${ }^{4}$

The positively skewed distribution of age of onset of EDs, including subthreshold presentations, renders adolescents a target for screening and early intervention before symptoms become severe and enduring, ${ }^{5}$ and research has supported that early intervention is associated with the most promising treatment outcomes. ${ }^{6}$ Increasing research has been devoted to the treatment of $\mathrm{AN}$ in adolescence, with an increasing number of studies published demonstrating considerable support for family-based treatment (FBT) approaches. ${ }^{7}$ To date, evidence to guide the treatment of $\mathrm{BN}$ in adolescents is limited, with only four published randomized controlled trials (RCTs) of psychological treatments and one open medication trial. ${ }^{8-11}$

\section{Prevalence}

Many population-based studies have focused on youths who meet full-threshold criteria for an ED without reporting on the cases who may have clinically significant presentations, but fall below the diagnostic threshold. ${ }^{1}$ In adults, the prevalence estimates of full-threshold $\mathrm{BN}$ are $1 \%-1.5 \%,{ }^{2,12}$ with $0.1 \%-2 \%$ in youth. ${ }^{13}$ However, community studies that evaluated disordered eating behavior rather than applying strict DSM criteria found far greater prevalence (ie, 14\%-22\%) than those applying strict criteria. ${ }^{1,14}$

In an attempt to estimate the occurrence of EDs in US adolescents better, Swanson et al utilized data from the National
Comorbidity Survey replication adolescent supplement. ${ }^{1}$ These data collected via face-to-face interviews included a representative sample of 10,123 youths aged 13-18 years. The World Health Organization Composite International Diagnostic Interview was used to assess DSM-IV psychiatric disorders, including $\mathrm{AN}, \mathrm{BN}$, and binge $\mathrm{ED}$, based on the diagnostic algorithms used with adult populations. Sufficient information was gathered to assess subthreshold presentations of AN using a less stringent weight criterion (ie, less than $90 \%$ of median weight for height rather than $85 \%$ ) and subthreshold binge ED. However, the survey did not include sufficient information to report on subthreshold presentations of DSM-IV BN, such as those who did not meet the duration or frequency criterion or those who experienced only subjective binge episodes. ${ }^{1}$

In the USA, the median age of onset for DSM-IV BN is quite young: 12.4 years (interquartile range $11.1-13.5$ years). ${ }^{1}$ Within the sample, the lifetime prevalence rate of $\mathrm{BN}$ was $0.9 \%$ and 12 -month prevalence $0.6 \%$. Of adolescents with BN, $41.3 \%$ reported purging, while the rest met criteria based on nonpurging compensatory behaviors (ie, excessive exercise, fasting). ${ }^{1}$ With revised diagnostic criteria reducing the frequency of binge eating and purging from twice per week over 6 months to once per week over 3 months, these numbers obviously underestimate the true prevalence of $\mathrm{BN}$ based on current criteria.

\section{Comorbidity}

The National Comorbidity Survey replication adolescent supplement also evaluated the presence of other DSM-IV diagnoses. The majority of respondents meeting criteria for a DSM ED also met criteria for another lifetime DSM-IV diagnosis. The highest rate of comorbidity ( $88 \%$ ) occurred in adolescents with $\mathrm{BN}$, with particularly strong associations with mood (49.9\%) and anxiety (66.2\%) disorders. ${ }^{1}$

Especially concerning is the level of suicidality among this group. Adolescents with BN reported suicidality at higher rates than adults with $\mathrm{BN}$ and higher rates than youth with any other ED diagnosis. Of adolescents with $\mathrm{BN}$, more than half (53\%) endorsed suicidal ideation. Over a quarter of the sample had a plan and more than a third had had a prior attempt (17.1\% with multiple past attempts). ${ }^{1,15}$

\section{Treatment for adolescent BN}

A diagnosis of $\mathrm{BN}$ is typically associated with high rates of comorbidity and elevated mortality. ${ }^{16}$ In addition, bingeeating and purging behaviors can lead to a range of medical complications, such as electrolyte imbalance, which can lead to arrhythmia or death, esophageal tears, gastric disruption, 
problems with fertility, and dental decay. ${ }^{17}$ With the risk of medical complications, it is imperative to utilize a multidisciplinary team approach, including a clinician who is trained in adolescent health with expertise in EDs to ensure the medical safety of outpatient care.

In adults with $\mathrm{BN}$, there is the substantial literature to support cognitive behavioral therapy (CBT) as the leading treatment approach, with relatively good outcomes for most. ${ }^{18}$ In addition to CBT, there is evidence to support other approaches, such as interpersonal therapy, ${ }^{18}$ and newer approaches, such as integrative cognitive-affective therapy. ${ }^{19}$ Despite the growing evidence base for treatment of adults with $\mathrm{BN}$, there remains a dearth of evidence for treating younger individuals with $\mathrm{BN}$. To date, there has been one open medication trial of fluoxetine in conjunction with therapy over the course of 8 weeks. There are also four published RCTs comparing psychosocial treatments with significant room for improvement of treatment outcomes.

\section{Psychopharmacological treatment}

One open clinical trial was published investigating the feasibility and tolerability of fluoxetine (at an adult dose of $60 \mathrm{mg}$ ) in conjunction with psychotherapy over the course of 8 weeks. ${ }^{20}$ Participants were female adolescents aged 12-18 (mean 16.2, SD 1) years with a DSM-IV diagnosis of BN or ED not otherwise specified (EDNOS). All participants were above the 85 th percentile of median weight for height by age..$^{20}$

The medication was generally well tolerated, and no participants discontinued the trial due to side effects. Of the 13 patients who entered the study, ten received a minimum of 1 week of medication, and results were based on their outcome. Findings were comparable to studies of fluoxetine in adults with $\mathrm{BN}$, with a significant decrease in both binge and purge behavior over the course of the trial. From baseline to the end of treatment (EOT), weekly binges decreased by $67 \%$ and episodes of purging by $56 \%$. Despite improvements, three of the ten patients continued to meet criteria for a DSM-IV ED (two BN and one EDNOS) at the end of the trial. ${ }^{20}$

These findings have not been replicated nor have there been any placebo-controlled trials in adolescents with BN. Therefore, the results remain limited in their generalizability, and additional exploration of psychopharmacological interventions is necessary.

\section{Psychological treatment}

Despite the typical onset during adolescence, there have been only four RCTs published to date evaluating psychosocial treatment for $\mathrm{BN}$ in youth. The first three compared FBT approaches against guided self-care CBT, CBT adapted for adolescence (CBT-A), and supportive psychotherapy (SPT). The most recently published study compared CBT and psychodynamic therapy (PDT).

\section{Family therapy and CBT-guided self-care}

Schmidt et al $^{10}$ were the first to publish findings from an RCT for the treatment of BN in adolescents. Their study, conducted in the UK, sought to compare the efficacy and cost-effectiveness of two treatments. Participants were 85 adolescents aged 13-20 years with DSM-IV BN or EDNOS. In the UK, guided self-care CBT is recommended as the firstline intervention for adults with BN. This treatment approach utilized a workbook and involved ten weekly sessions and three monthly follow-up sessions with the option of two sessions with a "close other" (eg, parent). The role of the therapist was to motivate the patients and guide them through the workbook. Similar to traditional CBT, self-monitoring was utilized, along with problem-solving and behavioral experiments. The comparison treatment was family therapy adapted from the Maudsley model of treatment for AN, as it has a growing evidence base for the treatment of young people with AN. This treatment provided psychoeducation about $\mathrm{BN}$ and was problem-oriented, with a focus on the role of the family in restoring regular eating patterns. In family therapy, patients were offered 13 sessions with a close other in addition to two individual sessions over the course of 6 months. $^{10}$

The primary outcome variable was abstinence from binge eating and vomiting in the month prior to assessment assessed at EOT (6 months) and follow-up (12 months). When looking at binge episodes and purging as a combined variable, there was no difference between conditions at either time point. However, CBT-guided self-care was more effective at achieving abstinence from objective binge eating (45\% versus $25 \%$ ) at EOT, but this advantage was not maintained at follow-up. The impact on purging did not vary by treatment. ${ }^{10}$

\section{FBT and SPT}

In the same year, Le Grange et $\mathrm{al}^{8}$ published another study comparing FBT and SPT. Participants were 80 youths ages 12-19 (mean 16.1, SD 1.6) years, predominantly female (98\%), with a diagnosis of DSM-IV BN or partial BN (ie, binge and purge episodes averaging once per week over 6 months). The duration of treatment was 20 sessions over the 
course of 6 months. Both treatments involved three phases, and the frequency of sessions decreases over time. ${ }^{8}$

FBT-BN was adapted from FBT for AN. This treatment takes an agnostic stance about the cause of the ED and assumes a significant negative impact of the illness on the family. FBT-BN also externalizes the illness as something separate from the adolescent, and empowers parents to disrupt the cycle of binge eating and purging. SPT is a nondirective treatment that does not involve specific active therapeutic approaches, such as stimulus control, psychoeducation, systematic problem-solving, or direct input about dietary modifications. SPT serves as a general treatment that might be comparable to what would be received in the community. ${ }^{8}$ The primary outcome measure was abstinence from binge eating and purging in the month prior to assessment at 6 months (EOT) and 12 months (follow-up). At EOT, FBT-BN had significantly higher rates of abstinence (39\% versus $18 \%$ ). However, the rate of abstinence declined during follow-up across both groups (29\% and $10 \%$, respectively) ${ }^{8}$

\section{FBT and CBT}

Following the comparison of FBT-BN to SPT, a control treatment similar to what youth with $\mathrm{BN}$ might receive in the community, Le Grange et al compared FBT-BN to CBT, which is the most efficacious approach for adults with BN. Participants were 109 youths aged 12-18 (mean 15.8, SD $1.5)$ years, predominantly female (94\%), with DSM-IV BN or partial BN (same as previously mentioned). Both treatments involved 18 sessions over the course of 6 months. ${ }^{9}$

FBT was comparable to the FBT-BN delivered in the previous study by Le Grange et al, ${ }^{8}$ aside from having two fewer sessions. CBT-A was adapted from the treatment developed by Fairburn et al. ${ }^{21}$ The treatment developed for adults was modified such that there was increased contact with the therapist in early treatment, with the goal of enhancing therapeutic alliance, collateral sessions with caregivers to provide psychoeducation about $\mathrm{BN}$ and elicit their support in the treatment process, the use of concrete examples to explain points, and the exploration of typical developmental issues. ${ }^{9}$

Consistently with the prior studies, the primary outcome was abstinence from binge eating and purging for the 4 weeks prior to assessment at EOT (6 months) and follow-up (12 months). The rate of abstinence was significantly higher for FBT-BN than CBT-A (39.4\% versus 19.7\%), with continued improvement at 6-month follow-up ( $44 \%$ and $25.4 \%$ respectively). ${ }^{9}$

\section{CBT and PDT}

In the most recently published RCT, Stefini et a ${ }^{11}$ compared CBT and PDT. In Germany, where the study was conducted,
$58.9 \%$ of psychotherapists for children and adolescents practiced PDT. This study sought to evaluate the gap in knowledge about commonly practiced PDT as a treatment for youth with BN. Participants in this sample were females aged 14-20 (mean 18.7, SD 1.9) years with a DSM-IV diagnosis of BN or partial BN (binge and purge episodes less than twice per week in previous 3 months).

The duration of the treatment was comparable to the guidelines of clinical practice in Germany, which involved substantially more treatment than the prior studies. Both CBT and PDT took place over 1 year, and participants could receive up to 60 sessions. Similar to Le Grange et al, ${ }^{9} \mathrm{CBT}$ was based on Fairburn et al's model of CBT for $\mathrm{EDs}^{22}$ with significant modifications, including tripling the number of sessions offered. PDT was manualized specifically for young people with BN. Both treatments share a symptom-focused approach that is specific to BN. However, underlying theoretical assumptions are quite different, and each approach places a different emphasis on emotions, cognitions, and behavior. ${ }^{11}$

Rather than evaluating abstinence from binge-eating and purging behavior 1 month prior to assessment, Stefini et al evaluated what proportion of participants no longer met the criteria for an ED and found no differences between groups, with 13 participants no longer meeting diagnostic criteria for an ED (33.3\% CBT and 30.2\% PDT). The rates of remission improved slightly, but not significantly for CBT (38.5\%) and were unchanged for PDT at follow-up 1 year after EOT. ${ }^{11}$

\section{Challenges in treatment}

The challenges previously discussed with diagnosis will ultimately impact the ability to screen effectively for EDs and connect with treatment. Shame and secrecy may prevent the detection of BN and may inhibit treatment after symptoms are identified. The family may serve a crucial role in enhanced support to regulate eating behavior between sessions. However, in BN, it may be less obvious when binge episodes or compensatory behavior is taking place, making it difficult for parents to structure the environment fully.

In $\mathrm{BN}$, the high level of comorbidity makes it probable that adolescents may present for treatment of another psychiatric disorder (eg, mood or anxiety disorder) while also struggling with binge eating and purging. Diagnostic assessment and ongoing monitoring of progress may offer opportunities to uncover clinically significant symptoms of BN. At the present time, there are limited data to guide the treatment of $\mathrm{BN}$ as a disorder in isolation, and there is no guidance about whether consecutive- or concurrent-treatment approaches for comorbid diagnosis are most appropriate or effective. 
That said, most RCTs to date have included participants with comorbid psychiatric diagnoses and provided pharmacotherapy for these diagnoses alongside psychosocial treatment for the ED. It also remains unclear why RCTs of treatments for $\mathrm{BN}$ within younger populations are lagging behind. Taken together, the limited systematic evaluation and concomitant advancement of treatment approaches for this patient population leaves us with a constrained scope of treatment options.

\section{Conclusion and future directions}

Despite the increasing number of RCTs for treatment of adolescents with AN and adults with BN, there continues to be few systematic studies of treatment of $\mathrm{BN}$ in adolescents. While FBT-BN has preliminary evidence to support the involvement of caregivers in treatment, there is considerable room for improvement to impact binge-eating and purging symptoms further among adolescent sufferers. In instances where caregivers are unable to participate in treatment or patients unwilling to assent to a high degree of parental involvement in their treatment, there is evidence that CBT approaches are helpful for some adolescents. The early study by Schmidt et a ${ }^{10}$ suggested that a stepped-care approach beginning with guided self-care may be sufficient for some, whereas other adolescents will ultimately require a more intensive level of treatment. PDT and CBT in an extended format (ie, up to 60 session over 1 year, rather than the more typical 20 sessions over 6 months) both decreased the number of individuals meeting criteria for full-threshold $\mathrm{BN}$.

Further research is necessary to enhance treatment approaches for adolescents with BN. While including caregivers in treatment shows promising results, there may be ways in which the cognitive symptoms can be more systematically addressed within the context of the family approach to treatment. It is also possible that family-based approaches might be enhanced with techniques in CBT, such as self-monitoring and behavioral experiments to challenge problematic beliefs. Further research is necessary to identify for whom which treatment approach will be most effective. In addition, adaptations of existing efficacious treatments or other heretofore unexplored treatment approaches should be examined, especially for those individuals who do not respond to the existing treatment approaches.

\section{Disclosure}

Dr. Le Grange receives royalties from Guilford Press and Routledge, and is co-director of the Training Institute for
Child and Adolescent Eating Disorders, LLC. The authors report no other conflicts of interest in this work.

\section{References}

1. Swanson SA, Crow S, Le Grange D, Swendsen J, Merikangas KR. Prevalence and correlates of eating disorders in adolescents: results from the national comorbidity survey replication adolescent supplement. Arch Gen Psychiatry. 2011;68(7):714-723.

2. American Psychiatric Association. Diagnostic and Statistical Manual of Mental Disorders. 5th ed. Washington, DC: APA; 2013.

3. American Psychiatric Association. Diagnostic and Statistical Manual of Mental Disorders. 4th ed, text revision. Washington, DC: APA; 2000.

4. Le Grange D, Loeb KL, Van Orman S, Jellar CC. Bulimia nervosa in adolescents: a disorder in evolution? Arch Pediatr Adolesc Med. 2004;158(5):478-482.

5. Le Grange D, Loeb KL. Early identification and treatment of eating disorders: prodrome to syndrome. Early Interv Psychiatry. 2007;1(1):27-39.

6. Treasure J, Russel G. The case for early intervention in anorexia nervosa: theoretical exploration of maintaining factors. Br J Psychiatry. 2011;199(1):5-7.

7. Murray SB, Le Grange D. Family therapy for adolescent eating disorders: an update. Curr Psychiatry Rep. 2014;16(5):447.

8. Le Grange D, Crosby RD, Rathouz PJ, Leventhal B. A randomized controlled comparison of family-based treatment and supportive psychotherapy for adolescent bulimia nervosa. Arch Gen Psychiatry. 2007;64(9):1049-1056.

9. Le Grange D, Lock J, Agras WS, Bryson SW, Jo B. Randomized clinical trial of family-based treatment and cognitive-behavioral therapy for adolescent bulimia nervosa. J Am Acad Child Adolesc Psychiatry. 2015;54(11):886-894.

10. Schmidt U, Lee S, Beecham J, et al. A randomized controlled trial of family therapy and cognitive behavior therapy guided self-care for adolescents with bulimia nervosa and related disorders. Am J Psychiatry. 2007:164(4):591-598.

11. Stefini A, Salzer S, Reich G, et al. Cognitive-behavioral and psychodynamic therapy in female adolescents with bulimia nervosa: a randomized controlled trial. J Am Acad Child Adolesc Psychiatry. 2017;56(4):329-335.

12. Hudson JI, Hiripi E, Pope HG Jr, Kessler RC. The prevalence and correlates of eating disorders in the national comorbidity survey replication. Biol Psychiatry. 2007;61(3):348-358.

13. Merikangas KR, He JP, Brody D, Fisher PW, Bourdon K, Koretz DS. Prevalence and treatment of mental disorders among US children in the 2001-2004 NHANES. Pediatrics. 2010;125(1):75-81.

14. Jones JM, Bennett S, Olmsted MP, Lawson ML, Rodin G. Disordered eating attitudes and behaviours in teenaged girls: a school-based study. CMAJ. 2001;165(5):547-552.

15. Crow SJ, Swanson SA, Le Grange D, Feig EH, Merikangas KR. Suicidal behavior in adolescents and adults with bulimia nervosa. Compr Psychiatry. 2014;55(7):1534-1539.

16. Arcelus J, Mitchell AJ, Wales J, Nielsen S. Mortality rates in patients with anorexia and other eating disorders: a meta-analysis of 36 studies. Arch Gen Psychiatry. 2011;68(7):724-731.

17. Mehler PS, Rylander M. Bulimia nervosa: medical complications. $J$ Eat Disord. 2015;3:12.

18. Agras WS, Walsh T, Fairburn CG, et al. A multicenter comparison of cognitive-behavioral therapy and interpersonal psychotherapy for bulimia nervosa. Arch Gen Psychiat. 2000;57(5):459-466.

19. Wonderlich SA, Peterson C, Crosby RD, et al. A randomized controlled comparison of integrative cognitive-affective therapy (ICAT) and cognitive-behavioral therapy-enhanced (CBT-E) for bulimia nervosa. Psychol Med. 2014;44(3):543-553.

20. Kotler L, Devlin MJ, Davies M, Walsh TB. An open trial of fluoxetine for adolescents with bulimia nervosa. J Child Adolesc Psychopharmacol. 2003;13(3):329-335. 
21. Fairburn CG, Marcus MD, Wilson GT. Cognitive-behavioral therapy for binge eating and bulimia nervosa: a comprehensive treatment manual. In: Fairburn CG, Wilson GT, editors. Binge Eating: Nature, Assessment, and Treatment. New York: Guilford Press; 1993:361-404.
22. Fairburn CG, Cooper Z, Shafran R. Enhanced cognitive behavior therapy for eating disorders (CBT-E): an overview. In Fairburn CG, editor, Cognitive Behavior Therapy and Eating Disorders. New York: Guilford Press; 2008:23-34.

\section{Publish your work in this journal}

Adolescent Health, Medicine and Therapeutics is an international, peer-reviewed, open access journal focusing on health, pathology, and treatment issues specific to the adolescent age group. All aspects of health maintenance, preventative measures and disease treatment interventions are addressed within the journal and practitioners from all disciplines are invited to submit their work as well as healthcare researchers and patient support groups. This journal is included in PubMed. The manuscript management system is completely online and includes a very quick and fair peer-review system. Visit http://www.dovepress.com/testimonials. php to read real quotes from published authors.

Submit your manuscript here: http://www.dovepress.com/adolescent-health-medicine-and-therapeutics-journal 